\title{
Optimized Clustering for Maximal Lifetime of Wireless Sensor Networks*
}

\author{
Kyung Tae Kim, Hyunsoo Kim, and Hee Yong Youn ${ }^{* *}$ \\ School of Information and Communication Engineering \\ Sungkyunkwan University, Suwon, Korea \\ \{harisu, hyunsoo\}@skku.edu, youn@ece.skku.ac.kr
}

\begin{abstract}
Wireless sensor network consisting of a large number of small sensors is efficient in gathering data in a variety of environments. Since the sensor nodes operate on batteries, energy efficient operations are indispensable to maximize the lifetime of the network. Among the schemes proposed to improve the lifetime of the network, the cluster-based schemes aim to evenly distribute the energy consumption among all the nodes in the network. In this paper we propose an approach for finding an optimal number of clusters which allows minimal energy consumption of the network. The key idea of the proposed approach is to model the energy consumption with independent homogeneous spatial Poisson process, while considering the distribution of cluster-heads and other sensor nodes. With the number of cluster-heads obtained by the proposed approach, the energy consumption can be significantly reduced and consequently the lifetime of the sensor network is increased compared to the existing schemes. Computer simulation confirms this with practical operational environment.
\end{abstract}

Keywords: Cluster-head, energy-efficiency, network lifetime, optimized clustering, wireless sensor networks.

\section{Introduction}

Wireless sensor network (WSN) consists of a large number of tiny sensor nodes forming an ad-hoc distributed sensing and data propagation network for collecting context information on the physical environment. It has been evolved rapidly for both military and civilian applications such as target tracking, surveillance, and security management $[1,2]$. A sensor node has four basic components: a sensing unit, a processing unit, a radio unit, and a power unit. One of the most restrictive factors regarding the lifetime of wireless sensor network is energy resource of the deployed sensor nodes. Because the sensor nodes carry limited and generally irreplaceable power sources, the protocols designed for WSN must seriously take the issue of energy efficiency into consideration. The clustering-based routing protocols proposed for the WSN try to evenly distribute the consumption of the energy of the sensors $[3-5,9]$. The main idea

\footnotetext{
* This research was supported by the Ubiquitous Autonomic Computing and Network Project, 21st Century Frontier R\&D Program in Korea and the Brain Korea 21 Project in 2005.

** Corresponding author.
} 
of the protocol is based on dynamical selection of the cluster-heads among the eligible active nodes.

In Low-Energy Adaptive Clustering Hierarchy (LEACH) [6], a communication protocol and its energy efficiency in homogenous networks are presented. LEACH is a clustering-based protocol employing the approach of randomized rotation of the cluster-heads to evenly distribute the energy load among the sensors in the network. Together with local data fusion, $\mathrm{LEACH}$ achieves a significant improvement in network lifetime compared with the Direct, MTE (Minimum Transmission Energy) [11], and Static Clustering approach. In $\mathrm{LEACH}$, it is assumed that every node has a packet to send to a distant base station in a round of communication. Proxy-Enabled Adaptive Clustering Hierarchy (PEACH) [7] adopts a similar approach as LEACH but proposes an additional scheme. It selects a proxy node which can assume the role of the current cluster-head of weak power during one round of communication. PEACH is based on the consensus of healthy nodes for the detection and manipulation of failure in any cluster-head.

In LEACH and PEACH, the cluster-based topology reduces long range communication, data fusion saves energy by compressing the data, and rotation of the clusterheads enables balanced energy consumption, which eventually prolong the lifetime of the nodes. The most important factor in the cluster-based protocol is the number of clusters in the network. In the previous protocols the number of clusters are fixed throughout the lifetime of the network regardless of the number of live nodes. It is, however, against the intuition that the number of clusters need to be decided in proportion to the number of live nodes.

In this paper we propose an approach finding the number of clusters which allows minimal consumption of the energy of the entire network. The key idea of the proposed approach is to model the energy consumption of the network with independent homogeneous spatial Poisson process, while considering the distribution of cluster-heads and other sensor nodes. With the number of cluster-heads obtained by the proposed approach, the energy consumption can be significantly reduced and consequently the lifetime of the sensor network is increased compared to the existing schemes. Computer simulation confirms this with practical operational environment.

The remainder of the paper is organized as follows. Section 2 presents the background of the problem addressed including the energy model. Section 3 introduces the proposed approach. Section 4 evaluates the performance of it by computer simulation, and compares it with LEACH and PEACH. Finally, Section 5 concludes the paper and outlines the future research directions.

\section{The Background}

In this paper we focus on a single-hop sensor network as in LEACH and PEACH. There exist three kinds of nodes in the WSN; sensor nodes, cluster-head nodes, and base station. Each sensor node is small and low-cost; they are used for data acquisition. The cluster-heads are for data fusion and forwarding of the aggregated data to the base station. As a result, the cluster-heads consume energy at a substantially higher rate than other nodes due to wireless communication over large distances. The 
base station may be assumed to always have sufficient battery power, or its battery may be reprovided during its course of operation. Therefore, its power consumption is not a concern in our investigation.

\subsection{The Energy Model of a Sensor}

We use the same radio model as discussed in [6, 8], which is the first order radio model. In this model the radio unit dissipates $50 \mathrm{~nJ} / \mathrm{bit}\left(E_{\text {elec }}\right)$ to run the transmitter or receiver circuitry and $100 \mathrm{pJ} / \mathrm{bit} / \mathrm{m}^{2}\left(\xi_{\mathrm{mp}}\right)$ for the transmitter amplifier. The energy consumption model is described as follows. When a node transmits $k$-bit data to another node of a distance of $d$, the energy it consumes is $E_{T x}(k, d)=E_{\text {elec }} \times k+\varepsilon_{\text {amp }}$ $\times k \times d^{2}$. When a node receives $k$-bit data, the energy it consumes is $E_{R x}(k)=E_{\text {elec }} \times k$. The second term of $E_{T x}$ covers the energy loss due to channel attenuation, while $\varepsilon_{m p}$ is the amplifier coefficient. For simplicity of calculation, we assume that transmission range of all the nodes are same on one condition that the range should cover all the neighbors in its cluster. We also assume that all data packets are same sizes. For fair comparison, we use the same constant coefficients adopted in LEACH and PEACH.

\subsection{The Problem Statement}

In LEACH, the cluster-heads are stochastically selected. For this each node determines a random number between 0 and 1 . If the number is smaller than a threshold, the node becomes a cluster-head for the current round. The threshold for node $n$ is set as follows:

$$
T(n)=\frac{P}{1-P \times\left(r \bmod \frac{1}{P}\right)} \quad, \forall n \in G: T(n)=0 \quad, \forall n \notin G
$$

with $P$ as probability for a node to be the cluster-head, $r$ as the sequence number of the current round, and $G$ as the set of nodes that have not been cluster-head yet in the last $1 / P$ rounds. This algorithm ensures that every node becomes a cluster-head exactly once within $1 / P$ rounds. In $\mathrm{LEACH}, P$ is a fixed value of 0.05 . However, $P$ might need to be varied because the number of live nodes varies, actually decreases. We thus find the optimal probability for a node to be a cluster-head in the next section.

Data transmission failure occurs when a cluster-head cannot transmit data due to energy deficiency. It affects the system operation and causes a remedial operation such as re-clustering or boot-strap which results in time waste and reduced network lifetime, etc. We assume that data in the communication is error-free, and the semantic-related generic faults in the data are detected and removed by application specific operation. Data transmission faults in a cluster-head can also be caused by hardware failure. They can prevent the cluster-head from transmitting data to the sensors as well as relaying the data to the base station. The data sent by the sensor nodes will be lost if a cluster-head fails. We call all such failures complete cluster-head failures because the cluster-head can no longer serve as a liaison between the sensor nodes and base station. 


\section{The Proposed Scheme}

In this section we introduce the proposed approach. The operation of the proposed scheme consists of rounds. Each round of communication consists of three phases; the phase for finding the optimal number of cluster-heads, set-up phase, and data collection and transmission phase. In the first phase, the optimal probability for a node to be a cluster-head is determined. In the setup phase, clusters are organized and a schedule is transmitted to the member nodes. During the data transmission phase, the sensor nodes transmit the sensed data to the cluster-heads which are then forwarded to the base station according to the preset schedule.

\subsection{The Optimal Cluster-Head Probability Decision Phase}

In a sensor network the expected distance between the cluster-heads to the base station and the expected distance between the sensors to the cluster-head in a cluster depend on the number of sensors, the number of clusters, and the size of the region where the network is deployed. The expected distance between a sensor and the cluster-head in a cluster decreases while the distance between the cluster-head and base station increases as the number of clusters increases in a bounded region. An opposite phenomenon is observed when the number of clusters decreases. Therefore, an optimal value of $p$ in terms of energy efficiency needs to be decided by properly taking account the tradeoff between sensor-to-cluster-head and cluster-head-to-base station communication overhead.

Let $S$ denote a bounded region of a plane and $X(S)$ does the number of sensors contained in $S$. Then $X(S)$ is a homogeneous spatial Poisson process if it distributes the Poisson postulates, yielding a probability distribution

$$
P\{X(S)=n\}=\frac{\left[\lambda A(S)^{n} \mathrm{e}^{-\lambda \mathrm{A}(\mathrm{S})}\right]}{n !}, \quad n=0,1,2 \ldots
$$

Here $\lambda$ is a positive constant called the intensity parameter of the process and $A(S)$ represents the area of region $S$.

If region $S$ is a square of side length, $M$, then the number of sensors in it follows a Poisson distribution with a mean of $\lambda A(S)$, where $A(S)$ is $M^{2}$. Assume that there exist $N$ sensors in the region for a particular realization of the process. If the probability of becoming a cluster-head is, $p$, then $N_{P}$ sensors will become cluster-heads on average. Let $D_{B}(x, y)$ be a random variable denoting the distance between a sensor located at $(x$, $y$ ) and the base station. Let $P_{S}$ be the probability of existence of sensors uniformly distributed in region $S$. Without loss of generality, we assume that the base station is located at the center of the square region (i.e. the origin coordinate). Then, the expected distance from the base station to the sensors is given by

$$
E\left[D_{B}(x, y) \mid X(S)=N\right]=\iint_{S} D_{B}(x, y) \cdot P_{S} d S=\int_{-M / 2}^{M / 2} \int_{-M / 2}^{M / 2} \sqrt{x^{2}+y^{2}} \frac{1}{M^{2}} d_{x} d y=0.3825 M
$$

Since there exist $N_{p}$ cluster-heads on average and location of a cluster-head is independent of those of other cluster-heads, the total length of the segments from all the cluster-heads to the base station is $0.3825 N_{p} M$. 
Since a sensor becomes a cluster-head with a probability $p$, we expect that clusterhead and other sensors are distributed in a cluster as an independent homogeneous spatial Poisson process. Each sensor joins the cluster of the closest cluster-head to form a cluster. Let $X(C)$ be the random variable denoting the number of sensors except the cluster-head in a cluster. Here, $C$ is the area of a cluster. Let $D_{C}$ be the distance between a sensor and the cluster-head in a cluster. Then, according to the results of [10], the expected number of non-cluster-heads in a cluster and the expected distance from a sensor to the cluster-head (assumed to be at the center of mass of the cluster) in a cluster are given by

$$
E\left[D_{C} \mid X(S)=N\right]=\iint_{C} \sqrt{x^{2}+y^{2}}(x, y) d A(C)=\int_{0}^{2 \pi} \int_{0}^{M / \sqrt{N p \pi}} r^{2} \frac{N p}{M^{2}} d r d \theta=\frac{2 M}{3 \sqrt{N p \pi}},
$$

respectively. Here, region $C$ is a circle with radius $M / \sqrt{N p \pi}$. The sensor density of the cluster, $k(x, y)$, is uniform, and it is approximately $M^{2} / N_{\mathrm{p}}$.

Let $E_{C}$ be the expected total energy used by the sensors in a cluster to transmit one unit of data to their respective cluster-head. Since there are $N_{p}$ clusters, the expected value of $E_{C}$ conditioned on $X(S)=N$ is given by,

$$
E\left[E_{C} \mid X(S)=N\right]=N(1-p) \cdot \frac{E\left[D_{C} \mid X(S)=N\right]}{r}=N p^{\frac{1}{2}} \cdot \frac{2 M}{3 r \sqrt{\pi}} \frac{1-p}{\sqrt{p}} .
$$

If the total energy spent by the cluster-heads to transmit the aggregated information to the base station is denoted by $E_{B}$, then

$$
E\left[E_{B} \mid X(S)=N\right]=N p \cdot \frac{E\left[D_{B} \mid X(S)=N\right]}{r}=\frac{0.3825 N p M}{r} .
$$

Let $E_{T}$ be the total energy consumption with the condition of $X(S)=N$ in the network. Then

$$
E\left[E_{T} \mid X(S)=N\right]=E\left[E_{C} \mid X(S)=N\right]+E\left[E_{B} \mid X(S)=N\right] .
$$

Taking the expectation of Equation (7), the total energy consumption of the network is

$$
E\left[E_{T}\right]=E\left[E\left[E_{T} \mid X(S)=N\right]\right]=E\left[X(S)^{1 / 2}\right] \cdot \frac{2 M}{3 r \sqrt{\pi}} \frac{1-p}{\sqrt{p}}+E[X(S)] \cdot \frac{0.3825 p M}{r},
$$

where $E[\cdot]$ is expectation of a homogeneous Poisson process.

$E\left[E_{T}\right]$ will have a minimum value for a value of $p$, which is obtained by the first derivative of Equation (8)

$$
2 c_{2} p^{3 / 2}-c_{1}(p+1)=0,
$$

where $c_{1}=2 M \cdot E\left[(X(S))^{1 / 2}\right]=3 \sqrt{\pi}$ and $c_{2}=0.3825 M \cdot E[X(S)]$.

Equation (9) has three roots, two of which are imaginary. The second derivative of Equation (8) is positive and log concave for the only real root of Equation (9), and hence the real root minimizes the total energy consumption, $E\left[E_{T}\right]$. 
The only real root of Equation (9) is as follows.

$$
\begin{aligned}
p_{\text {opt }}= & \frac{0.0833 c_{1}^{2}}{c_{2}^{2}}+\frac{0.1050\left(c_{1}^{4}+24 c_{1}^{2} c_{2}^{2}\right)}{c_{2}^{2}\left(2 c_{1}^{6}+72 c_{1}^{4} c_{2}^{2}+432 c_{1}^{2} c_{2}^{4}+83.1384 c_{1}^{2} \sqrt{c_{1}^{2} c_{2}^{6}+27 c_{2}^{8}}\right)^{1 / 3}} \\
& +\frac{0.0661}{c_{2}^{2}}+\left(2 c_{1}^{6}+72 c_{1}^{4} c_{2}^{2}+432 c_{1}^{2} c_{2}^{4}+83.1384 c_{1}^{2} \sqrt{c_{1}^{2} c_{2}^{6}+27 c_{2}^{8}}\right)^{1 / 3} .
\end{aligned}
$$

\subsection{The Set-Up Phase}

In the set-up phase, a portion of sensors stochastically elect themselves as clusterheads. For this the same process explained in Section 2.2 (Equation (4)) is executed, using $p_{\text {opt }}$ instead of $p$.

Each node determines a random number between 0 and 1 . If the number is smaller than a threshold, the node becomes a cluster-head for the current round. The threshold of node $n, T_{\text {new }}(n)$, is set as follows:

$$
\begin{gathered}
T_{\text {new }}(n)=\frac{p_{\text {opt }}}{1-p_{\text {opt }} \times\left(r \bmod \frac{1}{p_{\text {opt }}}\right)} \quad, \quad \forall n \in G \\
T_{\text {new }}(n)=0 \quad, \forall n \notin G
\end{gathered}
$$

with $p_{\text {opt }}$ of Equation (10), $r$ as the number of the current round, and $G$ as the set of nodes that have not been a cluster-head in the last $1 / p_{\text {opt }}$ rounds. This algorithm ensures that every node becomes a cluster-head exactly once within $1 / p_{\text {opt }}$ rounds.

The node electing itself as a cluster-head for the current round broadcasts an advertisement message to the rest of the nodes.

Section 3.1 has analyzed the problem of the population of cluster-head in wireless sensor network. It allows an optimal cluster-head probability for homogeneous network with various parameter settings. Cluster-heads are randomly selected using the optimal probability at the set-up phase. When a node is selected as a cluster-head, it generates a cluster-head token. Then, every selected cluster-head advertises its token by the CSMA/CA MAC protocol to all its neighbors. The noncluster-head nodes receive these advertisements and compare their signal strength. It keeps only the token with the strongest signal and randomly chooses a one when a tie occurs. After the advertisements, every non-cluster-head node recognizes the source of the token as its cluster-head and broadcasts the topology answer packet by the CSMA/CA MAC protocol back to the cluster-head. In the answer packet, the node's position (NP) and remaining energy (RE) level are included. When the cluster-heads receive the answer packets, they set up a schedule for its local cluster. Based on the number of nodes in the cluster, the cluster-head creates a TDMA schedule telling when each node can transmit the packets. The schedule is broadcast back to the nodes in the cluster. 


\subsection{The Data Collection and Transmission Phase}

After the set-up phase, the data collection and transmission phase starts. Every node collects local data, and sends the packet to the cluster-head in its allocated transmission time. Based on the strength of the received cluster-head advertisement signal and the assumption of symmetrical radio channel, the transmission can use a minimum amount of energy. The radio of other nodes are turned off until their allocated transmission time to save the energy. Each cluster-head keeps its receiver on to collect data from its member nodes and continuously updates the energy table in the schedule based on the received packets. When the data from all non-cluster-head nodes have been received, the cluster-heads process data fusion to aggregate all received data into one signal. The data fusion can be a simple averaging or complex data processing. After data fusion, the cluster-heads send the information of the cluster to the base station. As the cluster-heads need to receive many packets and consume large power for long range transmission, they are the ones whose energy is used up most quickly in the cluster. Therefore, a cluster-head can cause a failure due to energy deficiency. If a failure occurs at a cluster-head, the network has to be re-clustered and a new schedule needs to be transmitted to the sensors. This will reduce the network lifetime. In order to extend the lifetime of network by avoiding such problem, a proxy node is selected.

Calculation of the Threshold Value. The threshold value $E_{T H}$ plays a very important role in the data transmission phase since it is used as a measure for deciding if the current cluster-head has become obsolete. When the energy of a cluster-head drops below the threshold, the proxy node selection process begins. We assume that all sensors are identical and produce data at the same rate. The following functions are used for deciding the threshold value.

$$
\begin{gathered}
k_{j}=M_{b i t}\left[\frac{n}{k}\right]-1 \\
\sum_{i=1} k_{i j} \\
E_{C H(j)}=E_{\text {elec }} \times k_{j}+\varepsilon_{a m p} \times k_{j} \times d_{C H(j)}{ }^{2} \\
E_{T H}=\frac{1}{k} \sum_{j=1}^{k} E_{C H(j)}
\end{gathered}
$$

Here $k_{j}$ is the length of the aggregated message in the cluster-head and $d_{C H}$ is the distance between the cluster-head and base station. Since $E_{T H}$ changes over time, the threshold is calculated in every data collection and transmission phase.

When the energy level of the cluster-head falls below the threshold, data transmission is aborted and a proxy node is selected. It is done using the RE and NP value of the reply packets received in the set-up phase (Refer to [7]). After a node is selected as a proxy node, the cluster-head broadcasts an Indicator Control Message (ICM) containing the address of the proxy node and a new TDMA schedule to the member node. The member nodes that receive the ICM send a confirmation message to the proxy node, which includes the node ID of the member node. After the message exchange is over, the member nodes resume data transmission.

The proposed approach can reduce the error which can occur when the clusterheads of low energy transmit data to the base station. It will also evenly distribute 
energy consumption among the nodes in the network, and as a result increase lifetime of the entire sensor network. Also, it can raise the system reliability by reducing energy consumption and waste of time required for boot-strapping and set-up initialization involved in re-clustering.

\section{Performance Evaluation}

We evaluate the effectiveness of the proposed scheme along with Direct, LEACH, and PEACH through computer simulation. The probability for a node to be selected as a cluster-head is decided using the model developed in Section 3.1. For the simulation we consider a sensor network of 100 sensor nodes randomly located in a $50 \times 50$ region. A base station is located at the center $(25,25)$. We use two models of initial residual energy of sensor nodes; uniform at $0.5 \mathrm{~J}$ and random between $0.25 \mathrm{~J}$ and $0.5 \mathrm{~J}$. We set $E_{\text {elec }}$ to $50(\mathrm{~nJ} / \mathrm{bit})$ and $\xi_{m p}$ to $100\left(\mathrm{pJ} / \mathrm{bit} / \mathrm{m}^{2}\right)$ in the energy model of a sensor. The size of sensor data is 2000 bits, and the advertisement message is 64-bit long. In the simulation the result of 100,000 runs are averaged.

Table 1 lists the lifetime of the sensor network in terms of the round a node begins to die and the round the last node dies for the four schemes compared. Notice that the proposed scheme is consistently better than the others. Especially, the proposed scheme outperforms more significantly when the initial energy is relatively high. We ran the simulator with different energy thresholds, and obtained similar results.

Table 1. The network lifetimes with different initial energies of the sensors

\begin{tabular}{|c|c|c|c|}
\hline $\begin{array}{c}\text { Energy } \\
(\mathrm{J} / \text { node })\end{array}$ & Protocol & The round a node begins to die & The round a node begins to dies \\
\hline \multirow{3}{*}{0.25} & Direct & 85 & 141 \\
\cline { 2 - 4 } & LEACH & 312 & 596 \\
\cline { 2 - 4 } & PEACH & 367 & 683 \\
\cline { 2 - 4 } & Proposed & 423 & 812 \\
\hline \multirow{3}{*}{0.5} & Direct & 146 & 279 \\
\cline { 2 - 4 } & LEACH & 628 & 1012 \\
\cline { 2 - 4 } & PEACH & 782 & 1157 \\
\cline { 2 - 4 } & Proposed & 995 & 1364 \\
\hline
\end{tabular}

The improvement offered by the proposed scheme over LEACH and PEACH can be clearly seen in Figure 1, which shows the number of sensors alive as the round proceeds with the energy of $0.25 \mathrm{~J} /$ node initially. A sensor node with insufficient residual energy can occasionally become a cluster-head even though there is a sensor node of larger battery power nearby. It then exhausts the energy, stops operating, and disrupts the entire network operation. Also, data transmission to the base station is not possible. On the other hand, in the proposed scheme, the approach of proxy node and distribution of cluster-heads considering the distance to the base station allows significantly increased network lifetime. 


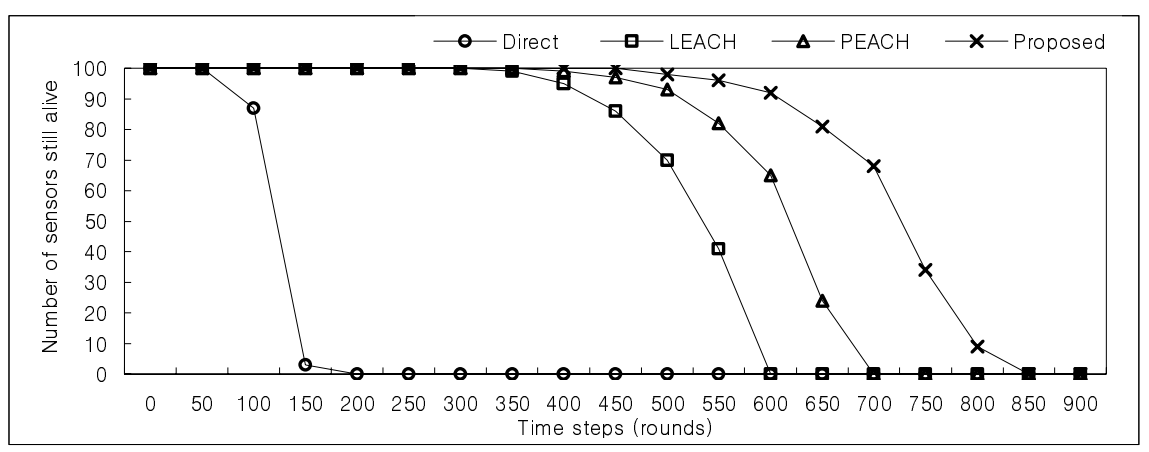

Fig. 1. The comparison of the number of live sensors as the round proceeds

Another important aspect of the proposed protocol is illustrated in Figure 2, which shows the locations of live (circle) and dead (dot) sensor nodes with LEACH and the proposed scheme, respectively, after 550 rounds. Observe that, in addition to a lot more live nodes than LEACH, the proposed scheme displays well dispersed live nodes. This is an important aspect that the proposed scheme can avoid dead spot while extending the lifetime of the network. This was achieved by employing an optimal number of clusters and proxy node for weak cluster-head.

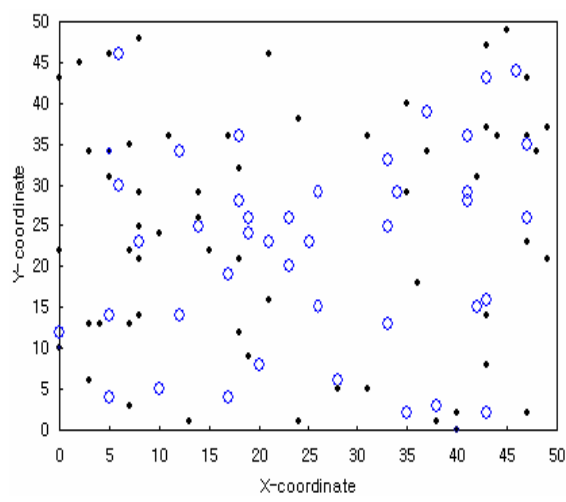

(a) $\mathrm{LEACH}$

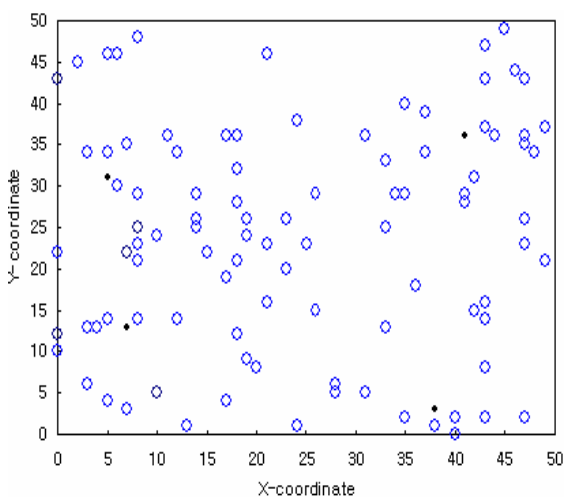

(b) The proposed scheme

Fig. 2. The distribution of live (circle) and dead (dot) nodes after 550 rounds

\section{Conclusion and Future Work}

In this paper we have proposed an approach for finding an optimal number of clusters which allows minimal energy consumption of the wireless sensor network. It is based on the modeling of energy consumption of the network with independent homogeneous spatial Poisson process, while considering the distribution of cluster-heads and other sensor nodes. The proposed approach allows considerable improvement in the 
stability of the system and reduces the overhead of re-clustering and system reconfiguration. Computer simulation showed that the proposed approach allows much longer lifetime of wireless sensor network than the existing schemes such as LEACH and PEACH. The proposed approach will be more important when the wireless sensor network is deployed in large area and the base station is far from the network.

The future work will focus on the comparison of the proposed approach with other approaches such as simulated annealing and taboos search. A formal methodology will also be developed in order to determine the factors used in each round of communication in a more systematic way and there by allow optimal results for the given condition. Our current simulation concentrates on one-hop cluster performance. It will be extended for energy-efficient data dissemination with multi-hop clusters.

\section{References}

[1] L. Zhong, R. Shah, C. Guo, J. Rabaey.: An ultra low power and distributed access protocol for broadband wireless sensor networks: IEEE Broadband Wireless Summit, Las Vegas, ay 2001.

[2] K. Sohrabi, J. Gao, V. Ailawadhi, and G. J. Pottie.: Protocols for self-organization of a wireless sensor network: IEEEPersonal Commun., 7(5):16-27, Oct. 2000.

[3] C.R. Lin and M. Gerla.: Adaptive Clustering for Mobile Wireless Network: IEEE J. Select. Area Commun, vol 15, pp. 1265-1275, Sept 1997.

[4] J. H. Ryu, S. Song, and D. H. Cho.: Energy-Conserving Clustering Scheme for Multicasting in Two-tier Mobile Ad-Hoc Networks: Elec-tron. Lett., vol. 37, pp. 1253-1255, Sept 2001.

[5] T. C. Hou and T. J. Tsai.: Distributed Clustering for Multimedia Support in Mobile Multihop Ad Hoc Network: IEICE Trans. Commun., vol. E84B, pp. 760-770, Apr 2001.

[6] W.R.Heinzelman, A.Chandrakasan, and H. Balakrishnan.: Energy-Efficient Communication Protocol for Wireless Micro-sensor Networks: In Proceedings of the Hawaii International Conference on System Science, Maui, Hawaii,2000.

[7] K.T. Kim and H.Y. Youn.: PEACH: Proxy-Enable Adaptive Clustering Hierarchy for Wireless Sensor network: Proceeding of The 2005 International Conference On Wireless Network, June 2005, pp. 52-57.

[8] W. Heinzelman, A. Chandrakasan, H. Balakrishnan.: An applicationspecific protocol architecture for wireless microsensor networks: in press IEEE Transaction on WirelessNetworking.

[9] P. Gupta and P.R. Kumar.: The capacity of wireless networks: IEEE Transaction on Information Theory, Vol. IT-46, No. 2, 388-404, March, 2000.

[10] S.G. Foss and S.A. Zuyev.: On a Voronoi Aggregative Process Related to a Bivariate Poisson Process: Advances in Applied Probability, Vol. 28, No. 4, pp. 965-981, 1996.

[11] T. Shepard.: A Channel Access Scheme for Large Dense Packet Radio Networks: In Proc. ACM SIGCOMM, pages 219-230, August 1996 